\title{
Oligodendrocyte Marker O4
}

National Cancer Institute

\section{Source}

National Cancer Institute. Oligodendrocyte Marker O4. NCI Thesaurus. Code C104202.

A sulfated galactosylceramide that is a differentiation marker on the surface of

oligodendrocyte precursors in the central nervous system. 